\title{
Medium size tokamak T-15MD as a base for experimental fusion research in Russian Federation
}

\author{
Alexander Romannikov and Fusion research Centre team
}

NRC “Kurchatov institute”, Moscow, Russia, Romannikov_AN@nrcki.ru

History of tokamak has more than 65 years. "Kurchatov institute" is a leader in Russia of high temperature plasma researches on tokamak facilities. It was the first in the world that has built the first tokamak and it is remained the world leader in researches on tokamaks for long time. At present time, the new tokamak T-15MD is being built in NRC "Kurchatov institute".

$\mathrm{T}-15 \mathrm{MD}$ main parameters are:

\begin{tabular}{lc}
\hline \multicolumn{1}{c}{ Parameter } & Value \\
\hline Major radius of torus R, $\mathrm{m}$ & 1.48 \\
Aspect ratio & 2.2 \\
Plasma current I, MA & 2.0 \\
Elongation, $\mathrm{k}$ & 1.9 \\
Triangularity, $\delta$ & $0.3-0.4$ \\
Plasma configuration & $\mathrm{SN}, \mathrm{DN}$ \\
Discharge pulse duration, s & 10 \\
Toroidal magnetic field at plasma axis, T & 2 \\
Magnetic flux swing in central solenoid, Wb & 6 \\
Neutral beam injection power, MW & 6 \\
ECR heating power, MW & 7 \\
ICR heating power, MW & 6 \\
LH heating power, MW & 4 \\
\hline
\end{tabular}

The magnetic system with divertor configuration of T-15MD facility for confining of hot plasma is already created. The toroidal magnet system consists of $16 \mathrm{D}$ shaped coils (Fig. 1) that form an arched structure [1-3].

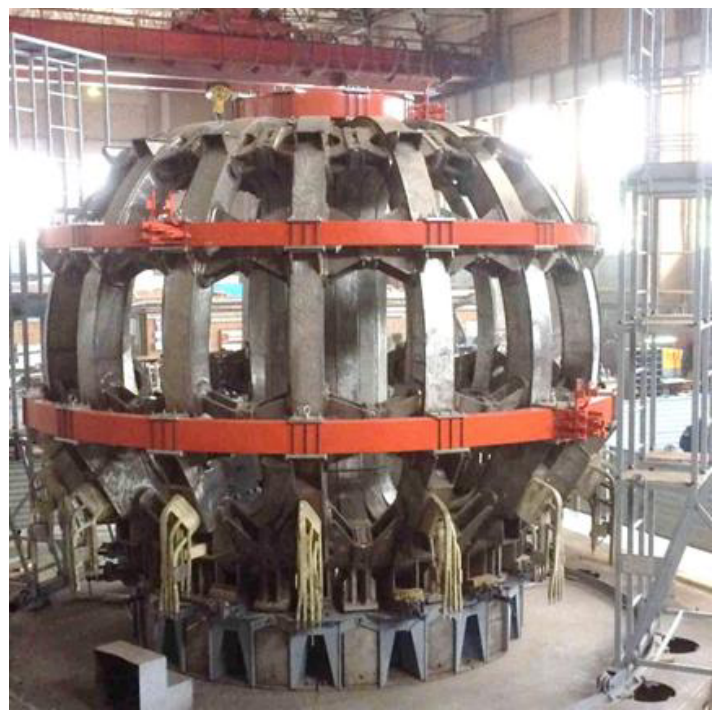

Fig. 1. Toroidal winding together with poloidal field coils

The level of ripples at the outboard plasma boundary is approximately $0.8 \%$. Each coil has 50 turns that are wound by a hollow conductor made of silver-copper al- loy. The toroidal winding is cooled with distilled water. Each coil has four parallel water branches. The total water mass flow rate through the winding is $43 \mathrm{~m}^{3} / \mathrm{h}$, with a pressure drop of $0.8 \mathrm{MPa}$.

The toroidal magnet is charged by eight thyristor convertors (22 kA, $1 \mathrm{kV}$ each). The rated value of the toroidal magnetic field at the plasma axis is $\mathrm{B}_{0}=2 \mathrm{~T}$. The current plateau duration is ten seconds.

The central solenoid (Fig. 2) consists of three separated coils. Each coil is wound with two parallel hollow conductors that have a trapezoidal form and are made of silver-copper alloy. The coils are charged by independent power supply systems.

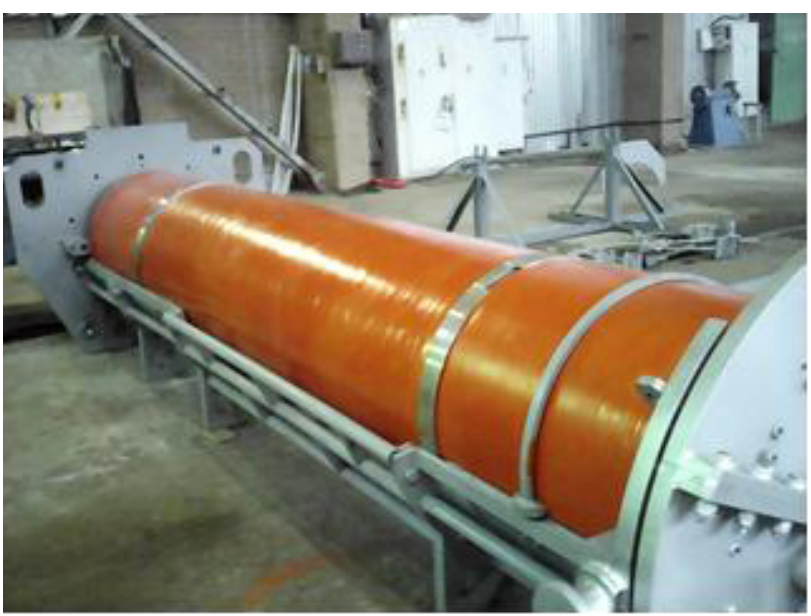

Fig. 2. Central solenoid

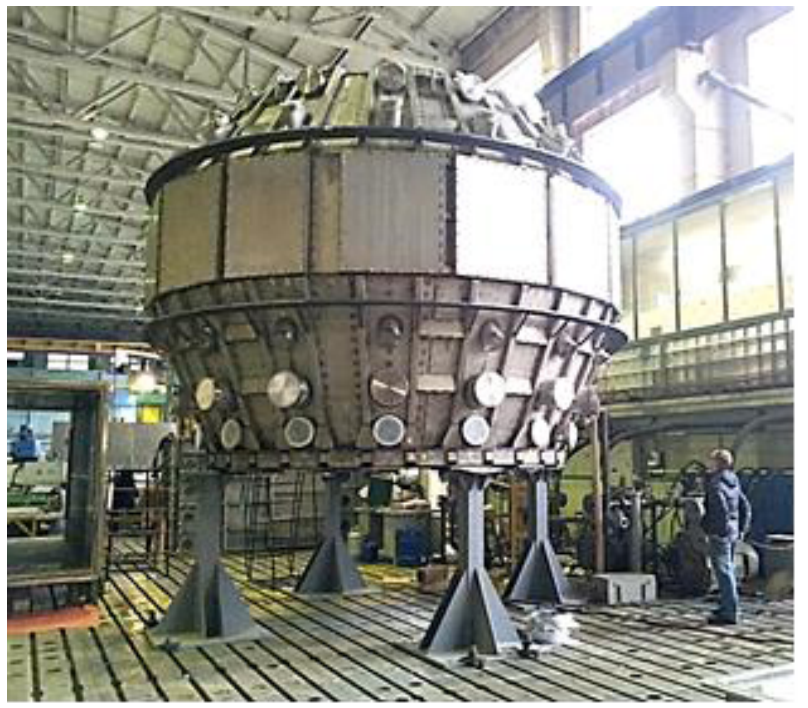

Fig. 3. Vacuum vessel shell

The next step of preassembly of the magnet system will be the integration of part of the vacuum vessel (Fig. 3) into toroidal winding. At the beginning the 
"cold" and "hot" vacuum tests of tokamak vacuum chamber will be carried out at the plant in Bryansk. After the preliminary assembling of the main tokamak systems will be carried out. For one vacuum vessel shell will be cut to four parts (four quarter sections). Half of the toroidal coils and two horizontal field coils will disassembled. Using the special rails two sectors of the vacuum vessel will be integrated inside the half of the toroidal winding. After finishing the preassembly of magnet system together with vacuum vessel parts, all elements will be disassembled and delivered to the Kurchatov Institute for assembly of the tokamak T-15MD in 2017-2018. The Tokamak T-15MD should begin operations in 2019.

The engineering systems [4] are important part of the overall system of the tokamak. The part of which is already ready now. They consist from:

1) High vacuum pumping system of the chamber with four turbopumps with total capacity of $\sim 10 \mathrm{~m}^{3} / \mathrm{s}$ $\left(\mathrm{H}_{2}\right)$ and two cryogenic pumps with the capacity of $4 \mathrm{~m}^{3} / \mathrm{s}$ $\left(\mathrm{H}_{2}\right)$ each.

2) Distiller water cooling system with a pressure of $0.5 \mathrm{MPa}$ and 1.0 $\mathrm{MPa}$. All equipment is installed and adjusted.

3) Power supply systems. The consumed total power in an impulse with current of plasma $2 \mathrm{MA}$ and additional heating of plasma of $20 \mathrm{MW}$ will make $300 \mathrm{MBA}$. Reconstruction of substations No. 745 and No. 110 (110/10 $\mathrm{kV}$ ) are planning to complete in 2017-2018.

4) Management information system [5] which is created as modular, scalable, with possibility of replication, the system focused on work of large experimental physical installation. Now the Data-processing center is created, installation of equipment of the main systems is carried out, tests and check of operability of some subsystems are carried out, commissioning for realization of physical start-up of installation in 2018-2019 is carried out.

The experimental program of $\mathrm{T}-15 \mathrm{MD}$ facility is based on experimental dates of T-10 tokamak achieved during a few last years. The main tasks of experimental program of T-15MD tokamak are: support of the ITER project in field of physical and technological database; development of tokamak fusion neutron source; fusion investigations in frame of "National Russian program on fusion and plasma technology" developing at present time.

More detailed of the experimental investigations program on the tokamak T-15MD includes the following tasks: the possibility of obtaining high values of beta as a way to reduce the cost of fusion reactor; research of plasma-materials interaction, including graphite, tungsten and lithium; monitoring of the plasma current and pressure profiles; research of the possibility of implementing a large value of beta and density in steady-state operations fully non-inductive current regimes; the control in real time of the stabilization, balance, heating and confinement the high temperature plasma, etc. Tokamak T-15MD will be used as a facility, which will be tested in the materials and technology of the first wall and divertor.

The installation is equipped with system of additional plasma heating and current drive $\left(\mathrm{P}_{\text {sum }}=15-20 \mathrm{MW}\right)$, which will allow one simultaneous the achievement of high temperature plasma $\left(\mathrm{T}_{\mathrm{i}} \sim \mathrm{T}_{\mathrm{e}} \sim 5-9 \mathrm{keV}\right)$ and plasma density (ne $\sim 10^{20} \mathrm{~m}^{-3}$ ) in the discharge with pulse duration up to $30 \mathrm{~s}$. This system involves the injection of neutrals ( 3 injector on $2 \mathrm{MW} / 75 \mathrm{keV} \mathrm{H} \mathrm{H}^{0}$, each), electroncyclotron heating (7 gyrotrons of 1.0-1.5 MW each, $f=110-120 \mathrm{GHz}$ with the possibility of heating at the second harmonic, and Bershtein's modes), ion cyclotron heating (3 antennas on $2 \mathrm{MW}$ each, including the possibility of maintaining the current helicons), lower hybrid heating and current drive (grill with a power of $4 \mathrm{MW}$, $f=2.45 \mathrm{GHz}$.

\section{References}

1. Bondarchuk, E.N., Azizov E.A., Alekseev A.B., et al. Engineering Problems of Tokamak T-15 Electromagnet System Reconstruction // IEEE Transactions on Applied Superconductivity, 2012, Volume: 22 , Issue: 3, Page(s): 4201604.

2. Khvostenko P.P., Azizov E.A., Alfimov D.E. et al. The magnet system of the Tokamak T-15 upgrade // Fusion Engineering and Design, 2015, vol. 98-99, 1090-1093.

3. Khvostenko P.P., Anashkin I.O., Belyakov V.A., et al. Preassembly of the tokamak T-15MD magnet system // Fusion Engineering and Design, 2017, http://doi.org/10.1016/j.fusengdes.2017.03.064

4. P.P. Khvostenko, I.O. Anashkin, V.A. Belyakov, et al. Status of Tokamak T-15MD // 26th IAEA Fusion Energy Conference, 17-22 October 2016, Kyoto, Japan // Book of abstracts, FIP/P7-40, p.749; eproceedings, fec2016-preprints, preprint0289.pdf

5. Motckin Y.Ya., Sokolov M.M., Khvostenko P.P. Information and control system for experimental fusion device "Tokamak T-15" // MKA : BKC, 2015, №3, 29-37. 